\title{
Helmholtz solitons in power-law optical materials
}

\author{
J. M. Christian, G. S. McDonald and R. J. Potton \\ Joule Physics Laboratory, School of Computing, Science and Engineering, \\ Institute for Materials Research, University of Salford, Salford M5 4WT, U.K.

\section{P. Chamorro-Posada} \\ Departamento de Teoría de la Señal y Comunicaciones e Ingeniería Telemática, \\ Universidad de Valladolid, ETSI Telecomunicación, Campus Miguel Delibes s/n, \\ 47011 Valladolid, Spain
}

\section{Accepted for Physical Review A (6 Sept 2007)}

A nonlinear Helmholtz equation for optical materials with regimes of power-law type of nonlinearity is proposed. This model captures broad beam evolution at any angle with respect to the reference direction in a wide range of media, including some semiconductors, doped glasses and liquid crystals. Novel exact analytical soliton solutions are presented for a generic nonlinearity, within which known Kerr solitons comprise a subset. Three new general conservation laws are also reported. Analysis and numerical simulations examine the stability of the Helmholtz powerlaw solitons. A new propagation feature, associated with spatial solitons in powerlaw media, constituting a new class of oscillatory solution, is identified.

PACS number(s): 42.65.Tg (optical solitons), 94.05.Fg (solitons and solitary waves), 05.45.Yv (solitons, nonlinear dynamics of). 


\section{INTRODUCTION}

Spatial solitons are well-known in nonlinear optics and have been studied for many years [1-3]. They are robust, localized nonlinear waves exhibiting self-stabilizing and self-guiding properties. Their remarkable stability under perturbation can be seen, for example, in the pair-wise collision between two solitons. They can exhibit mutual transparency, passing through each other elastically (no change in shape or velocity) and inducing only a trajectory phase shift (a lateral displacement in the position of each soliton centre from its unperturbed path). These features make spatial solitons ideal candidates for use in future Information Communication and Technology device applications [4-8]. Before such devices can be realized, it is necessary to have a thorough understanding of the interplay between diffraction and medium nonlinearity, and also of the limitations of conventional paraxial modelling.

The term 'nonparaxial' is often used to refer to ultranarrow or subwavelength optical beams [9-14], where the transverse waist $w_{0}$ and carrier wavelength $\lambda$ are comparable. However, a "nonparaxial = ultranarrow" interpretation is insufficiently general. In its widest sense, 'nonparaxial' means 'not paraxial' and refers to any situation where the paraxial approximation is violated. A beam may be described as "paraxial" if it is (i) broad compared to the carrier wavelength, (ii) of moderate intensity, and (iii) propagating in (or at a negligible angle with respect to) the reference direction. If all three criteria are not met simultaneously then the beam is, by definition, nonparaxial. Here we are concerned with the Helmholtz scenario, where conditions (i) and (ii) are always met rigorously but condition (iii) is relaxed. For completeness, the physical and mathematical character of ultranarrow-beam and Helmholtz contexts will now be discussed. 
Ultranarrow-beam nonparaxiality was effectively introduced by Lax et al. [9], who analysed the fully-vectorial Maxwell equations in terms of a single parameter-ofsmallness $\varepsilon \equiv \lambda / w_{0}$. It is now well-known that when $\varepsilon \sim O(1)$, transverse spatial variations of the electric field on the $\lambda$-scale lead to appreciable divergence in the nonlinear polarization. These steep gradients tend to produce strong coupling between components of the field. In such cases, the evolution of the dominant transverse component can be well described through an order-of-magnitude analysis of Maxwell's equations and retaining terms up to $O\left(\varepsilon^{2}\right)$. The governing equation turns out to be of the nonlinear Schrödinger (NLS) type, augmented by a range of higher-order diffractive corrections [9-14].

Helmholtz nonparaxiality is concerned with off-axis effects [15]. It differs fundamentally from ultranarrow-beam contexts, and the arbitrary-angle aspects of optical propagation cannot be captured by $\varepsilon$-type order-of-magnitude analyses. Indeed, it will be shown that the potentially dominant Helmholtz contribution to evolution is geometrical and can be of any order irrespective of $\varepsilon$. Here, we consider broad beams in two-dimensional planar waveguides that comprise a reference longitudinal direction $(z)$ and a single effective transverse direction $(x)$. In uniform media, $x$ and $z$ are physically indistinguishable and this spatial symmetry is respected by Helmholtz diffraction [16]. The explicit assumption of broad beams means that $\varepsilon \ll O(1)$ and the polarization-scrambling term $\nabla(\nabla \cdot \mathbf{E})$ in Maxwell's equations [914] is unimportant. One may treat the associated refractive-index distributions within the scalar approximation, and the electric field as a TE (transverse electric) polarized mode. 
In Helmholtz soliton theory [15], the governing equation is of the nonlinear Helmholtz (NLH) type. The spatial coordinates appear on an equal footing and diffraction is fully two-dimensional, occurring in both $x$ and $z$. By omitting the slowly-varying envelope approximation (SVEA), the angular restriction inherent to paraxial models is lifted and propagation may occur at any angle with respect to the reference direction. For Kerr media, where the refractive index varies with the square of the (local) optical field amplitude, exact analytical soliton solutions are now known $[15,17]$. Extensive numerical simulations have confirmed that they are stable robust entities surrounded by wide basins of attraction.

The power-law nonlinearity is of fundamental interest in optics [18]. It is perhaps the simplest generalization of the ubiquitous Kerr law and models a material whose refractive index depends on the optical field amplitude raised to a power other than 2. Various semiconductors, for example InSb [19] and GaAs/GaAlAs [20], doped filter glasses (such as $\mathrm{CdS}_{x} \mathrm{Se}_{1-x}$ ) [21], and liquid crystals (such as MBBA) [18], can possess power-law behaviour in their refractive index. Power-law solitary waves have been investigated theoretically in the context of interface surface modes [22], and as elementary excitations in thin films [23] and slab waveguides [24]. Snyder and Mitchell have also derived an exact soliton solution to a power-law NLS equation that describes the paraxial evolution of TE self-guided modes of a planar waveguide [25].

In this Article, we consider the broad range of optical materials whose fielddependent refractive-index distributions can possess power-law characteristics. In Section II, we propose a novel NLH governing equation with a power-law nonlinearity that captures the Kerr response as a particular case. The geometrical aspects of beam propagation are discussed and three new conservation laws reported. 
Two novel exact analytical soliton families are then presented and the structure of these solutions is explored in detail. In Section III, the advantages of using an elliptic evolution equation are reviewed and the stability of the new Helmholtz solitons is investigated both analytically and numerically. Perturbed non-Kerr power-law solitons are discovered to have quite different behaviour, under perturbation, from their Kerr subset. Conclusions are presented in Section IV.

\section{HELMHOLTZ POWER-LAW SOLITONS}

\section{A. Model equation}

We consider a continuous-wave scalar electric field with angular frequency $\omega$,

$$
\tilde{E}(x, z, t)=E(x, z) \exp (-i \omega t)+E^{*}(x, z) \exp (+i \omega t),
$$

propagating in a uniform planar waveguide. When the complex spatial envelope containing the field oscillations is assumed to vary on a scalelength much larger than $\lambda$, as it must for the scalar approximation to hold $[10,16], E(x, z)$ satisfies the NLH equation [15],

$$
\left(\frac{\partial^{2}}{\partial z^{2}}+\frac{\partial^{2}}{\partial x^{2}}\right) E(x, z)+\frac{\omega^{2} n^{2}}{c^{2}} E(x, z)=0 .
$$

This 'broad beam' model follows directly from Maxwell's equations when nonlinear divergence is neglected. The power-law nonlinearity is introduced through a refractive-index distribution $n(|E|)=n_{0}+n_{q}|E|^{q}$, where $n_{0}$ is the linear index, $n_{q}$ is a nonlinear coefficient and the exponent may assume continuum values $q>0$ [25]. The Kerr effect corresponds to $q=2$, and the generalized form also provides a model for saturable media when $q<2$ [22]. If $\left|n_{q}\right||E|^{q} \ll n_{0}$, which is usually satisfied for weak optical nonlinearities [2,3], then one has to an excellent approximation 
$n^{2} \simeq n_{0}^{2}+2 n_{0} n_{q}|E|^{q}$. To facilitate a comparison between Helmholtz and conventional (i.e. paraxial) models, the $z$ axis is chosen as the reference direction and the spatial part of the electric field is expressed as $E(x, z)=E_{0} u(x, z) \exp (i k z)$, where $k=n_{0} k_{0}$ and $k_{0} \equiv \omega / c=2 \pi / \lambda$. Using Eq. (2), one may derive the normalized equation for the envelope $u$ :

$$
\kappa \frac{\partial^{2} u}{\partial \zeta^{2}}+i \frac{\partial u}{\partial \zeta}+\frac{1}{2} \frac{\partial^{2} u}{\partial \xi^{2}} \pm|u|^{q} u=0
$$

The spatial coordinates are $\zeta=z / L_{D}$ and $\xi=\sqrt{2} x / w_{0}$, where $L_{D}=k w_{0}^{2} / 2$ is the diffraction length of a reference Gaussian beam with waist $w_{0}$. The \pm sign flags a focusing/defocusing nonlinearity, respectively, $E_{0}=\left(n_{0} / k\left|n_{q}\right| L_{D}\right)^{1 / q} \quad$ and $\kappa=1 / k^{2} w_{0}^{2} \equiv \varepsilon^{2} / 4 \pi^{2} n_{0}^{2} \ll O(1)$ quantifies the (inverse) beam width. Equation (3) has the three associated conserved quantities,

$$
\begin{gathered}
W=\int_{-\infty}^{+\infty} d \xi\left[|u|^{2}-i \kappa\left(u^{*} \frac{\partial u}{\partial \zeta}-u \frac{\partial u^{*}}{\partial \zeta}\right)\right], \\
M=\int_{-\infty}^{+\infty} d \xi\left[\frac{i}{2}\left(u^{*} \frac{\partial u}{\partial \xi}-\frac{\partial u^{*}}{\partial \xi} u\right)-\kappa\left(\frac{\partial u^{*}}{\partial \zeta} \frac{\partial u}{\partial \xi}+\frac{\partial u^{*}}{\partial \xi} \frac{\partial u}{\partial \zeta}\right)\right],
\end{gathered}
$$

and

$$
H=\int_{-\infty}^{+\infty} d \xi\left[\frac{1}{2} \frac{\partial u}{\partial \xi} \frac{\partial u^{*}}{\partial \xi}-\kappa \frac{\partial u}{\partial \zeta} \frac{\partial u^{*}}{\partial \zeta}-\frac{1}{1+\frac{1}{2} q}|u|^{2+q}\right]
$$

that represent the energy-flow, momentum and Hamiltonian, respectively. Conservation laws are of fundamental importance in physical systems, and integrals (4) can be used to monitor the integrity of the numerical scheme [26] used to solve Eq. (3). 
When analysing beam propagation in uniform media, a fundamental symmetry of the governing equation should be rotational invariance. This property follows directly from the fact that one has complete freedom to choose any orientation for the $(x, z)$ coordinate axes, relative to the beam [15]. For instance, if a beam is stable when the propagation and $z$ axes are parallel, it must also be stable when there is an arbitrary angle $\theta$ between them. One should expect this intuitively since the physical properties of the beam must be frame-independent. The SVEA breaks rotational invariance, limiting $\theta$ to vanishingly-small values only [15].

One should also recognize that even the simplest experimental arrangements can possess intrinsically angular characters that are outside the remit of the paraxial approximation. Two important examples are beam multiplexing and interface geometries. We have recently analysed these configurations for Kerr media using Helmholtz soliton theory [27,28]. New qualitative phenomena were uncovered in angular regimes, and corrections to paraxial theory in excess of $100 \%$ were predicted. The analysis of arbitrary-angle interaction/interface geometries that involve non-Kerr power-law materials cannot proceed without first having detailed knowledge of the corresponding exact analytical Helmholtz solitons.

\section{B. Exact Helmholtz solitons}

Since model (3) is second-order in the longitudinal coordinate, one expects to find both forward- and backward-propagating solutions. We have derived two exact analytical bright soliton solutions for a focusing nonlinearity, that are given by 


$$
\begin{gathered}
u(\xi, \zeta)=\eta \operatorname{sech}^{2 / q}\left(\frac{a(\xi \pm V \zeta)}{\sqrt{1+2 \kappa V^{2}}}\right) \\
\times \exp \left[i \sqrt{\frac{1+4 \kappa \beta}{1+2 \kappa V^{2}}}\left(\mp V \xi \pm \frac{\zeta}{2 \kappa}\right)\right] \exp \left(-i \frac{\zeta}{2 \kappa}\right), \\
a \equiv q\left(\frac{\eta^{q}}{2+q}\right)^{1 / 2},
\end{gathered}
$$

and $\beta \equiv 2 \eta^{q} /(2+q)$. Here, $\eta$ is the amplitude parameter and $V$ is the conventional transverse velocity parameter. The forward solution (upper signs) describes an exponentially-localized beam evolving with respect to the $+z$ axis at an angle $\theta=\tan ^{-1}(\sqrt{2 \kappa} V)$, where $-90^{\circ} \leq \theta \leq+90^{\circ}$, and $\theta>0$ is defined in an anti-clockwise sense [21]. The forward and backward solutions in (5), depicted in Fig. 1, can be combined into a single soliton whose propagation direction is determined solely by $\theta$ :

$$
\begin{aligned}
u(\xi, \zeta)=\eta \operatorname{sech}^{2 / q}\left[a\left[\xi \cos \theta+\frac{\zeta}{\sqrt{2 \kappa}} \sin \theta\right)\right] \\
\times \exp \left[i \sqrt{\frac{1+4 \kappa \beta}{2 \kappa}}\left(-\xi \sin \theta+\frac{\zeta}{\sqrt{2 \kappa}} \cos \theta\right)\right] \exp \left(-i \frac{\zeta}{2 \kappa}\right) .
\end{aligned}
$$

The profile of solitons (5) and (6) captures the angular beam-broadening factor $\left(1+2 \kappa V^{2}\right)^{-1 / 2}=\cos \theta$ (see Fig. 2), that can be non-negligible even in moderate-angle regimes. For instance, when $\theta= \pm 60^{\circ}$ an observer in the $(x, z)$ frame perceives the beam width to have doubled compared to its on-axis value [22]. The origin of the relation $\tan ^{2} \theta=2 \kappa V^{2}$ thus lies in $x-z$ equivalence, where the full generality of the $\partial_{z z}$ operator has been retained. Importantly, $2 \kappa V^{2}$ can be of any order of magnitude as $\theta \rightarrow \pm 90^{\circ}$, independently of the system nonlinearity, and even though $\kappa \ll O(1)$. At $\theta= \pm 90^{\circ}$, where $2 \kappa V^{2} \rightarrow \infty$, one finds that 


$$
u(\xi, \zeta)=\eta \operatorname{sech}^{2 / q}\left(a \frac{\zeta}{\sqrt{2 \kappa}}\right) \exp \left[\mp i \sqrt{\frac{1+4 \kappa \beta}{2 \kappa} \xi}\right] \exp \left(-i \frac{\zeta}{2 \kappa}\right)
$$

and the beam thus appears to be infinitely broad in $\xi$. Transforming to the $(x, z)$ frame $[21,43]$, it can be seen that (7) describes a soliton beam propagating in the $\mp x$ direction, respectively (i.e. perpendicularly to the $z$ axis). These two results (infinite width in $\xi$ and evolution along $\mp x$ ) are physically consistent with each other. We note that there is no analogue of (7) in paraxial theory.

Helmholtz solitons possess a range of generic features that arise from spatial symmetry. These features have no counterpart in paraxial theory, and include angular and intensity-dependent corrections to the beam wavevector, and the explicit appearance of the longitudinal phase term $\exp (-i k z)$. The absence of this factor from the paraxial solutions [25] prevents one from transforming rigorously between the $(\xi, \zeta)$ and $(x, z)$ coordinate frames.

For the new power-law solitons (5), it is possible to evaluate the conserved quantities analytically for arbitrary values of the exponent $q$.

$$
\begin{gathered}
W= \pm(1+4 \kappa \beta)^{1 / 2} P, \\
M=\frac{V}{\sqrt{1+2 \kappa V^{2}}}[(1+4 \kappa \beta) P \mp 2 \kappa Q], \\
H=\frac{W}{2 \kappa}-\left(\frac{1}{2 \kappa}\right) \frac{1}{\sqrt{1+2 \kappa V^{2}}}[(1+4 \kappa \beta) P-2 \kappa Q],
\end{gathered}
$$

where the upper (lower) signs denote the invariants of the forward (backward) beam. The additional parameters $P$ and $Q$ that appear in Eqs. (8) are given by

$$
P=\frac{\eta^{2}}{a} \gamma\left(2 q^{-1}\right)
$$




$$
Q=\left(\frac{2}{q}\right)^{2}\left(\frac{\eta^{2}}{a}\right)\left[\gamma\left(2 q^{-1}\right)-\gamma\left(2 q^{-1}+1\right)\right]
$$

and

$$
\gamma(y) \equiv \frac{\Gamma\left(\frac{1}{2}\right) \Gamma(y)}{\Gamma\left(\frac{1}{2}+y\right)}
$$

where $\Gamma$ is the gamma function. Analysis of Eqs. (8b) and (8c) reveals that for the forward soliton, the energy-momentum relationship $\partial H / \partial M=\partial_{V} H / \partial_{V} M=V$ holds, where $\partial_{V}$ denotes the derivative with respect to velocity $V$. New results for paraxial solitons appear as particular cases of Eqs. (8) and (9).

\section{Recovery of paraxial solitons}

It would be erroneous to conclude that the Helmholtz operator $\kappa \partial_{\zeta \zeta}$ can be omitted from Eq. (3) whenever $\kappa \ll O(1)$. The arbitrary magnitude of the term $2 \kappa V^{2}$ demonstrates that angular effects cannot generally be captured by ultranarrow-beam (i.e. single-parameter $\kappa$-type) corrections. Compelling evidence of the multi-fold character of $\kappa \partial_{\zeta \zeta}$ can be found, for example, in attempts to recover Synder and Mitchell's paraxial soliton [25] from (5); this cannot be achieved simply by setting $\kappa=0$. Instead, recovery is possible if and only if $(a) \kappa \rightarrow 0$ (broad beams), $(b)$ $\kappa \eta^{q} \rightarrow 0$ (moderate intensities) and (c) $\kappa V^{2} \rightarrow 0$ (negligible propagation angles, strictly $\left.\theta \rightarrow 0^{\circ}\right)$. This simultaneous multiple limit, which is an algebraic statement of $\kappa \partial_{\zeta \zeta} \rightarrow 0$, defines the paraxial approximation. When applied to the forward Helmholtz soliton, one obtains

$$
u(\xi, \zeta) \simeq \eta \operatorname{sech}^{2 / q}[a(\xi+V \zeta)] \exp \left[-i V \xi+i\left(\beta-\frac{V^{2}}{2}\right) \zeta\right]
$$


That is, the paraxial limit maps a forward Helmholtz beam onto its NLS counterpart. While (a) represents the scalar approximation, and $(b)$ is equivalent to $n_{0} \gg 2\left|n_{q}\right| E_{0}^{q}$ (i.e. the weak-nonlinearity approximation implicit in the derivation of both Eq. (3) and the corresponding paraxial equation), condition $(c)$ is a geometrical contribution that depends solely upon the choice of reference frame [15]. Interestingly, the fact that convergence of the Helmholtz beam to the paraxial solution requires $\theta \rightarrow 0^{\circ}$ (and does not occur for $\theta \rightarrow \pm 180^{\circ}$ ) emphasises the absence of backward waves in paraxial theory [25]. We also mention that by applying the multiple limit to Eqs. (8), one finds the paraxial conserved quantities, namely $W \simeq P, M \simeq V P$ and $H \simeq \frac{1}{2} V^{2} P-\beta P+Q$. Thus, $P$ is identical to the beam power of the corresponding paraxial soliton.

The elimination of $\kappa \partial_{\zeta \zeta}$ from conventional narrow-beam models must be carried out with care. In particular, one should ensure that all angular effects in the unscaled system can be safely neglected. Approximating $\kappa \partial_{\zeta \zeta}$ by a perturbation series (for example, in $\partial_{\xi \xi}$ operators $[10,29]$ ) destroys the bi-directionality of the governing equation. The resulting model is then parabolic, rendering finite-angle regimes inaccessible.

\section{STABILITY OF POWER-LAW SOLITONS}

Preserving the full generality of $\partial_{z z}$ allows Eqs. (2) and (3) to support forward and backward waves, so propagation may occur at any angle with respect to the reference direction. Ellipticity is thus a key feature of both models. Stable propagation in elliptic models has been known for several years [26]. A linear stability analysis reveals that Helmholtz and paraxial plane waves, in materials with 
arbitrary dispersive nonlinearity, are modulationally stable in the same parameter regimes [30]. For the power-law nonlinearity [25], plane waves with intensity $I_{0}$ possess a region of modulational instability in the long-wave spectral domain $\left|K_{\xi}\right|<\sqrt{2 q} I_{0}^{q / 4}$, where $K_{\xi}$ is the transverse wavenumber of the perturbation. We also mention that numerical simulations confirm excellent agreement between the predictions made by NLH-type models, such as Eq. (3), and those of nonlinear Maxwell equations [31].

\section{A. Analytical predictions}

Spatial symmetry allows one to analyse the stability of Helmholtz solitons using the well-known Vakhitov-Kolokolov (VK) integral criterion [2,32]. By rotating the $(x, z)$ coordinate axes so that the reference and propagation directions coincide, an isolated Helmholtz beam with $\kappa \ll O(1)$ and $\eta=O(1)$ in this "on-axis" frame of reference can be regarded as quasi-paraxial. The VK criterion states that a localized soliton can be stable against small perturbations if $d P / d \beta>0$, where $P$ is the beam power and $\beta$ is the longitudinal wavenumber. From Eq. (9a), it is straightforward to show that

$$
\frac{d P}{d \beta}=\frac{1}{2^{2 / q-1 / 2}} \gamma\left(\frac{1}{2} q\right) \frac{(2+q)^{2 / q}}{q}\left(\frac{2}{q}-\frac{1}{2}\right) \beta^{2 / q-3 / 2}
$$

From inspection of Eq. (11a), it can be seen that the slope of $P(\beta)$ is always positive provided $q<4$; when this inequality is met, $P(\beta)$ increases monotonically. The character of soliton stability is often connected to the curvature of $P(\beta)$ [33]. For power-law nonlinearity, 


$$
\frac{d^{2} P}{d \beta^{2}}=\frac{1}{2^{2 / q-1 / 2}} \gamma\left(\frac{1}{2} q\right) \frac{(2+q)^{2 / q}}{q}\left(\frac{2}{q}-\frac{1}{2}\right)\left(\frac{2}{q}-\frac{3}{2}\right) \beta^{2 / q-5 / 2} .
$$

The curvature is positive when $q<q_{\text {crit }}$, zero when $q=q_{\text {crit }}=\frac{4}{3}$, and negative when $q_{\text {crit }}<q<4$ (see Fig. 3). The existence of such a critical point (characterized by a change in the sign of $\left.d^{2} P / d \beta^{2}\right)$ suggests that one should expect a qualitative change in the behaviour of a perturbed soliton when $q \approx q_{\text {crit }}$.

\section{B. Numerical perturbative analysis}

We now evaluate the robustness of the new power-law Helmholtz solitons (5) against perturbations to their shape, through consideration of the initial condition

$$
u(\xi, 0)=\operatorname{sech}^{2 / q}(a \xi) \exp \left(-i V \sqrt{\frac{1+4 \kappa \beta}{1+2 \kappa V^{2}}} \xi\right)
$$

where $\beta=2 /(2+q)$. The input beam corresponds to a perturbed canonical (i.e. $\eta=1)$ soliton that is launched at an angle $\theta=\tan ^{-1}(\sqrt{2 \kappa} V)$ relative to the reference direction. Through a rotational transformation, it can be seen that (12) is entirely equivalent to an on-axis Helmholtz beam whose width has been reduced by a factor of $\left(1+2 \kappa V^{2}\right)^{1 / 2}$. Results are presented for a range of launching angles, $\theta=10^{\circ}, 30^{\circ}$ and $50^{\circ}$, which represent weak, moderate, and strong perturbations, respectively. These angles are clearly non-trivial, and lie outside the remit of the paraxial approximation. When $\kappa=10^{-3}\left(\kappa=10^{-4}\right)$, the transverse velocities are $V \approx 3.94$ $(V \approx 12.47), V \approx 12.91(V \approx 40.82)$, and $V \approx 26.65(V \approx 84.27)$, respectively.

When $\theta$ deviates from zero, self-reshaping oscillations appear in the parameters (amplitude, width, and area $=$ amplitude $\times$ width) of the evolving beam. 
The nature of these oscillations depends upon the nonlinearity exponent $q$. For $q=1$, sustained self-oscillation dominates the long-term evolution, and a stationary state does not appear to emerge as $\zeta \rightarrow \infty$. Over propagation lengths longer than those shown in Fig. 4(a), the reshaping oscillations are modulated by a slowly-varying envelope function.

For $q=2$, it is known that the reshaping oscillations strictly vanish as $\zeta \rightarrow \infty$ to leave a stationary beam [see Fig. 4(b)]. Thus, in Kerr media, one finds that the input beam can transform asymptotically into an exact Helmholtz soliton [34]. For quasi-paraxial beams, the properties of this asymptotic Helmholtz Kerr soliton can be predicted by combining geometrical considerations with inverse-scattering perturbation techniques [35]. For $q=3$, small perturbations tend to give rise to sustained self-oscillation in the beam parameters, similar to the behaviour found in the case of $q=1$, but of generally longer period. However, as the perturbation increases, self-focusing is insufficient to balance initial diffractive spreading. The peak amplitude decreases monotonically with distance, and the beam loses its solitonic properties [see Fig. 4(c)].

\section{Representation of perturbed solitons}

One way of representing the evolving beam is in the $\left(|u|_{m}, \partial_{\zeta}|u|_{m}\right)$ plane (see Fig. 5). The trajectories associated with perturbed $q=1$ solitons can then be associated with quasi-periodic orbits. For $q=2$, where the reshaping oscillations vanish asymptotically, the trajectory winds on to a fixed point with $\partial_{\zeta}|u|_{m}=\partial_{\zeta}\left(\partial_{\zeta}|u|_{m}\right)=0$ as $\zeta \rightarrow \infty$. This fixed point represents a stationary Helmholtz soliton, and its precise location on the $|u|_{m}$ axis depends upon the initial 
perturbation. We have classified the Helmholtz solitons with $q=2$ as fixed point attractors, and those with $q=1$ as limit cycle attractors [30]. Helmholtz solitons with $q=3$ are conditionally stable. These designations arise from the similarity between the phase portraits in Fig. 4 and those found in other nonlinear dynamical systems [36]. The fixed-point and limit-cycle terminology has been discussed in more detail elsewhere [30].

The stability properties of Helmholtz solitons are mapped in Fig. 6, as a function of nonlinearity index $q$ and the launching angle $\theta$ (that determines the magnitude of the perturbation). When $0<q<\frac{4}{3}$, a perturbed beam exhibits limitcycle reshaping oscillations, while for $\frac{4}{3}<q<\frac{5}{2}$ one observes fixed-point oscillations. The existence and nature of the bifurcation point at $q_{\text {crit }}=\frac{4}{3}$, predicted by the VK criterion, has thus been confirmed numerically. Numerical analysis has also identified a second bifurcation, occurring at $q=\frac{5}{2}$, at which point the reshaping oscillations revert from the fixed-point type back to limit-cycle type.

The existence of the second bifurcation point was not predicted from examining the $P(\beta)$ curves, which show no particular feature at $q=\frac{5}{2}$. Nonlinear analysis $[2,33,37]$ will inevitably be required to quantify this bifurcation further, and also to describe fully the internal mode-type oscillations uncovered in numerical simulations [33,37]. However, we find that the single (arbitrary) power-law introduces new complexities into the nonlinear analyses used earlier [33,37]. Moreover, stability analysis of NLH models also presents further complications, such as the inclusion of backward waves, and the fact that two initial conditions are required to solve elliptic equations. For example, the $\kappa \partial_{\zeta \zeta}$ operator hinders the decoupling of the real and imaginary parts of the perturbation field. Such decoupling 
allows solution of linearized eigenvalue problems associated with paraxial governing equations $[2,38,39]$.

\section{CONCLUSIONS}

A novel NLH equation describing optical beam propagation in a wide range of power-law materials [18-25] has been presented. Novel exact analytical forward- and backward-propagating bright soliton solutions have been derived, for which known Kerr solitons [15] are obtained when $q=2$. Ellipticity of the governing equation is an essential feature if one is to describe beam evolution [34], interaction [27] and interface [28] regimes involving oblique angles. The analysis of such scenarios in general power-law media requires a detailed knowledge of exact Helmholtz solitons (5) and also the corresponding conservation laws (8), both of which have been reported here for the first time. The geometrical properties of beam propagation have been explored in detail, and known paraxial solutions emerge from the new Helmholtz solutions in an entirely physical multiple limit.

Arbitrary-angle regimes are outside the scope of classic paraxial models, and it has been shown analytically that $\kappa \partial_{\zeta \zeta}$ can be strongly perturbative in off-axis configurations. Exact Helmholtz power-law solitons (5) have been found to be robust entities that propagate stably over arbitrarily long distances when $0<q<4$. Analysis and simulations have led directly to the identification of a new class of oscillatory solution associated with perturbed Helmholtz solitons in power-law media with $0<q<\frac{4}{3}$ and $\frac{5}{2}<q<4$. These oscillatory solutions have no counterpart in Kerr $(q=2)$ media. Our stability results also have implications for paraxial solitons. 
For completeness, we comment on the behaviour of the predicted exact Helmholtz soliton (5) when $q \geq 4$. It is well known that, in this regime, the powerlaw NLS model predicts an unphysical collapse of a localized beam to zero transverse size and infinite amplitude [2,25,39]. Numerical analysis reveals that this type of "blow up" can be suppressed in Eq. (3) and that, instead of such singular behaviour, the beam tends to undergo smooth diffractive spreading toward a zero-amplitude state (see Fig. 7). We thus find that an instability is also present in the $q \geq 4$ power-law Helmholtz model, but that the character of this instability may be profoundly different from that of its paraxial counterpart (where the contribution from $\kappa \partial_{\zeta \zeta}$ is neglected). This phenomenon is of interest in terms of universal amplitude equations involving Helmholtz-type generalization of the linear wave operator. However, consideration of higher-order nonparaxial effects is likely to be necessary for a full investigation of this phenomenon in the specific context of nonlinear optical beams.

The considerations in this Paper are of fundamental physical and mathematical interest, examining the structure and stability of novel exact solitons of non-integrable elliptic wave equations. Helmholtz soliton theory is proposed as essential for the accurate modelling of non-trivial angular contexts in nonlinear optics, and implications are expected for a wide range of experimental regimes.

\section{ACKNOWLEDGEMENTS}

The authors gratefully acknowledge enlightening discussions with Professor William J. Firth. 


\section{REFERENCES}

[1] G. Stegeman and M. Segev, Science 286, 1518 (1999).

[2] Y. S. Kivshar, Opt. Quant. Elec. 30, 571 (1998).

[3] Y. S. Kivshar and B. Luther Davies, Phys. Rep. 298, 81 (1998).

[4] T. T. Shi and S. Chi, Opt. Lett. 15, 1123 (1990).

[5] B. Luther-Davies and Y. Xiaoping, Opt. Lett. 17, 496 (1992).

[6] C. Anastassiou, M. Segev, K. Steiglitz, J. A. Giordmaine, M. Mitchell, M. Shih, S. Lan and J. Martin, Phys. Rev. Lett. 83, 2332 (1999).

[7] G. S. McDonald and W. J. Firth, J. Opt. Soc. Am. B 7, 1328 (1990).

[8] G. S. McDonald and W. J. Firth, J. Opt. Soc. Am. B 6, 1081 (1993).

[9] M. Lax, W. H. Louisell and W. B. McKnight, Phys. Rev. A 11, 1365 (1975).

[10] S. Chi and Q. Guo, Opt. Lett. 20, 1598 (1995).

[11] A. Ciattoni et al., Opt. Exp. 14, 5517 (2006); ibid J. Opt. Soc. Am. B 17, 809 $(2000)$

[12] A. Ciattoni, B. Crosignani, S. Mookherjea and A. Yariv, Opt. Lett. 30, 516 (2005); B. Crosignani, A. Yariv and S. Mookherjea, Opt. Lett. 29, 1524 (2004).

[13] E. Granot et al., Opt. Commun. 178, 431 (2000); ibid Opt. Lett. 22, 1290 (1997).

[14] B. A. Malomed, K. Marinov, D. I. Pushkarov and A. Shivarova, Phys. Rev. A 64, 023814 (2001); B. V. Gisin and B. A. Malomed, J. Opt. Soc. Am. B 18, $1356(2001)$.

[15] P. Chamorro-Posada, G. S. McDonald and G. H. C. New, J. Opt. Soc. Am. B 19, $1216(2002)$.

[16] M. D. Feit and J. A. Fleck, J. Opt. Soc. Am. B 5, 633 (1988). 
[17] P. Chamorro-Posada and G. S. McDonald, Opt. Lett. 28, 825 (2003).

[18] D. Mihalache, M. Bertolotti and C. Sibilia, Progress in Optics, 27, 227 (1989).

[19] J. G. H. Mathew, A. K. Kar, N. R. Heckenberg and I. Galbraith, IEEE J. Quantum Electron. QE-21, 94 (1985).

[20] D. S. Chemla, D. A. B. Miller and P. W. Smith, Opt. Eng. 24, 556 (1985).

[21] S. S. Yao et al., Appl. Phys. Lett. 46, 801 (1985); R. K. Jain and R. C. Lind, J. Opt. Soc. Am. 73, 647 (1983).

[22] A. W. Snyder and H. T. Tran, Opt. Commun. 98, 309 (1993); G. I. Stegeman et al. Opt. Commun. 56, 365 (1986).

[23] C. T. Seaton et al., IEEE J. Quantum Electron. QE-21, 774 (1985).

[24] L. Wu, Opt. Commun. 224, 51 (2003); G. I. Stegeman et al. IEEE J. Quantum Electron. QE-22, 977 (1986); U. Langbein, F. Lederer and H. Ponath, Opt. Commun. 53, 417 (1985).

[25] A.W. Snyder and D. J. Mitchell, Opt. Lett. 18, 101 (1993).

[26] P. Chamorro-Posada, G. S. McDonald and G. H. C. New, Opt. Commun. 192, $1(2001)$.

[27] P. Chamorro-Posada and G. S. McDonald, Phys. Rev. E 74, 036609 (2006).

[28] J. Sánchez-Curto, P. Chamorro-Posada and G. S. McDonald, Opt. Lett. 32, $1126(2007)$.

[29] S. Blair, Chaos 10, 570 (2000); A. P. Sheppard and M. Haelterman, Opt. Lett. 23, 1820 (1998); G. Fibich, Phys. Rev. Lett. 76, 4365 (1996).

[30] J. M. Christian, G. S. McDonald and P. Chamorro-Posada, J. Phys. A: Math. Theor. 40, 1545 (2007); ibid. 8601 (2007).

[31] P. Chamorro-Posada, J. Sánchez-Curto, J. M. Christian and G. S. McDonald, "From Maxwell's Equations to New Families of Helmholtz Solitons," in 
Proceedings of the $2^{\text {nd }}$ International Conference on Advanced Optoelectronics and Lasers, Vol. II: 108-112 (2005).

[32] M. G. Vakhitov and A. A. Kolokolov, Radiophys. Quantum Electron. 16, 783 (1973).

[33] D. Pelinovski, V. V. Afansjev and Y. Kivshar, Phys. Rev. E 53, 1940 (1996).

[34] P. Chamorro-Posada, G. S. McDonald and G. H. C. New, J. Mod. Opt. 47, 1877 (2000).

[35] J. Satsuma and N. Yajima, Suppl. Prog. Theor. Phys. 55, 284 (1974).

[36] C. Zhou, X. T. He and S. Chen, Phys. Rev. A 46, 2277 (1992).

[37] D. V. Skryabin, J. Opt. Soc. Am. B 19, 529 (2002), ibid. Phys. Rev. E 64, 055601 (2001).

[38] E. W. Laedke, K. H. Spatschek and L. Stenflo, J. Math. Phys. 24, 2764 (1983).

[39] Y. V. Katyshev, N. V. Makhaldiani and V. G. Makhankov, Phys. Lett. 66A, 456 (1978); E. W. Laedke and K. H. Spatschek, Phys. Lett. 74A, 205 (1979). 


\section{FIGURE CAPTIONS}

FIG. 1. Geometry of the (a) forward and (b) backward soliton solutions of Eq. (5), given by the upper and lower choice of signs, respectively. The propagation angle in both solutions has been defined so that $\theta>0$ is always measured in an anti-clockwise sense relative to the $+z$ direction.

FIG. 2. Angular beam broadening of $q=3$ Helmholtz solitons (4) for $\theta=0^{\circ}$ (solid line), $\theta=30^{\circ}$ (dashed line), $\theta=45^{\circ}$ (dotted line) and $\theta=60^{\circ}$ (dot-dash line, where the beam width has doubled relative to its on-axis value). The solid line represents the paraxial solution (7), where broadening is absent and the beam has the same width irrespective of the transverse velocity $V$.

FIG. 3. Beam power $P$ as a function of the parameter $\beta$ (the longitudinal phase in the corresponding paraxial model [25]), obtained from Eq. (9a). When $q<q_{\text {crit }}$, the curvature is positive, $d^{2} P / d \beta^{2}>0$. For $q=q_{\text {crit }}=\frac{4}{3}, P$ vs. $\beta$ is a straight line $\left(d^{2} P / d \beta^{2}=0\right)$. When $q_{c r i t}<q<4$, the curvature is negative $\left(d^{2} P / d \beta^{2}<0\right)$. 
FIG. 4. Universal reshaping oscillations in the peak amplitude $|u|_{m}$ for initial condition (12) when (a) $q=1$, (b) $q=2$, and (c) $q=3$. Part (b) corresponds to the reshaping of Kerr solitons [32]. Solid curves: $\theta=10^{\circ}$, dashed curves: $\theta=30^{\circ}$, dotdash curves: $\theta=50^{\circ}$. As $\zeta \rightarrow \infty$, the oscillations in (b) are, strictly, vanishing. This is not the case for the other two $q$ values, and the oscillations present survive in the long-term evolution.

FIG. 5. (a) Phase plane for Kerr solitons, where $q=2$. Perturbed initial conditions lead to trajectories that slowly spiral on to a node (stable fixed point) in the phase plane. This asymptotic soliton state has well-defined propagation-invariant parameters (i.e. amplitude and width). (b) Trajectories for the $q=1$ power-law nonlinearity wind on to a slowly-varying orbit that is characterized by quasi-periodic parameters.

FIG. 6. Schematic diagram illustrating the classification of canonical $(\eta=1)$ soliton stability characteristics depending upon the nonlinearity index $q$.

FIG. 5. Diffractive instability of the Helmholtz soliton (4) when $q=4.2$ for four different values of the nonparaxial parameter $\kappa$. Solid: $\kappa=10^{-3}$; dashed: $\kappa=0.5 \times 10^{-3}$; dotted: $\kappa=10^{-4}$; dot-dash: $\kappa=0.5 \times 10^{-4}$. The self-focusing "blow up" singularity of the corresponding paraxial (parabolic) model is suppressed by the Helmholtz operator $\kappa \partial_{\zeta \zeta}$, even when $\kappa \simeq 0$. 
FIGURE 1

J. M. Christian et al.

to appear: Physical Review A

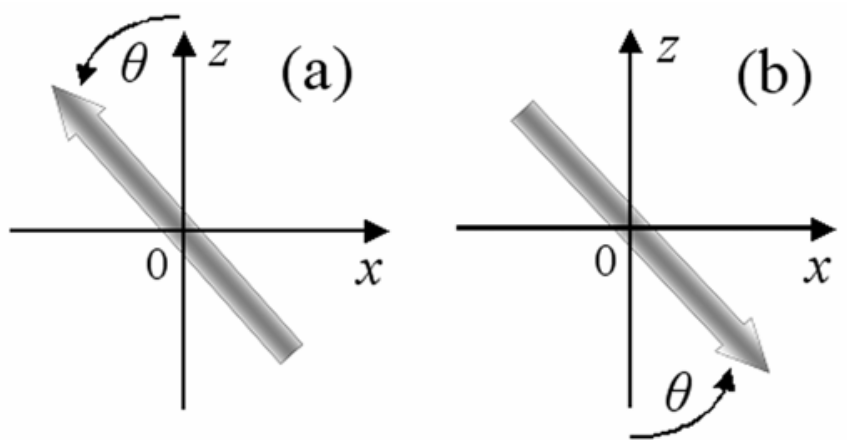


FIGURE 2

J. M. Christian et al.

to appear: Physical Review A

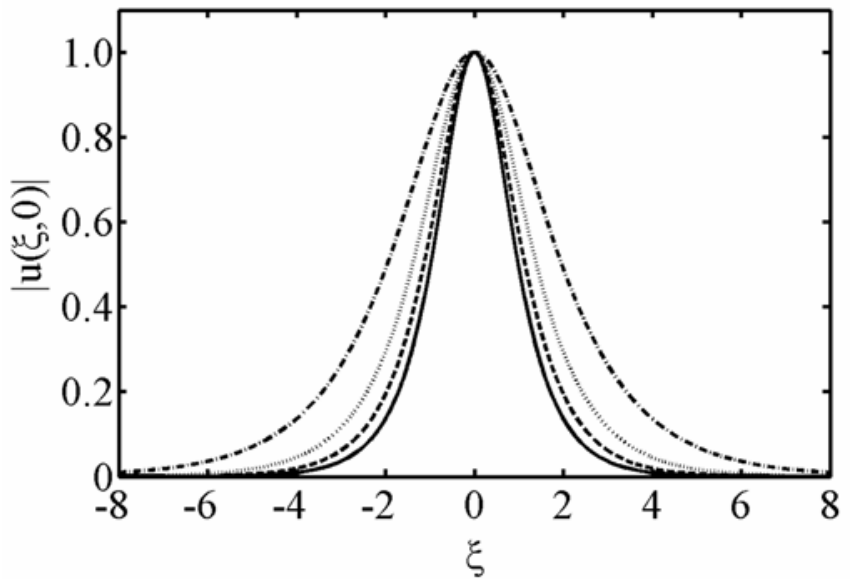


FIGURE 3

J. M. Christian et al.

to appear: Physical Review A

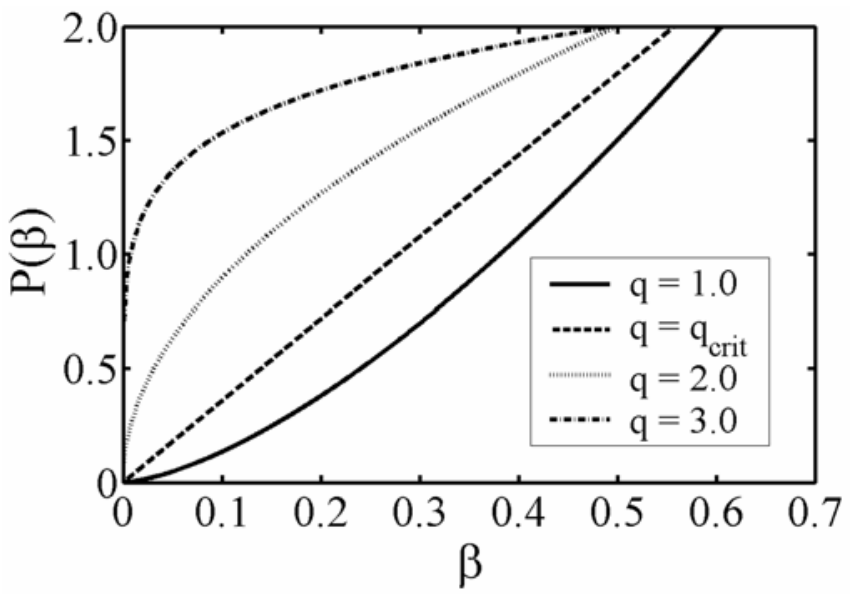


FIGURE 4

J. M. Christian et al.

to appear: Physical Review A
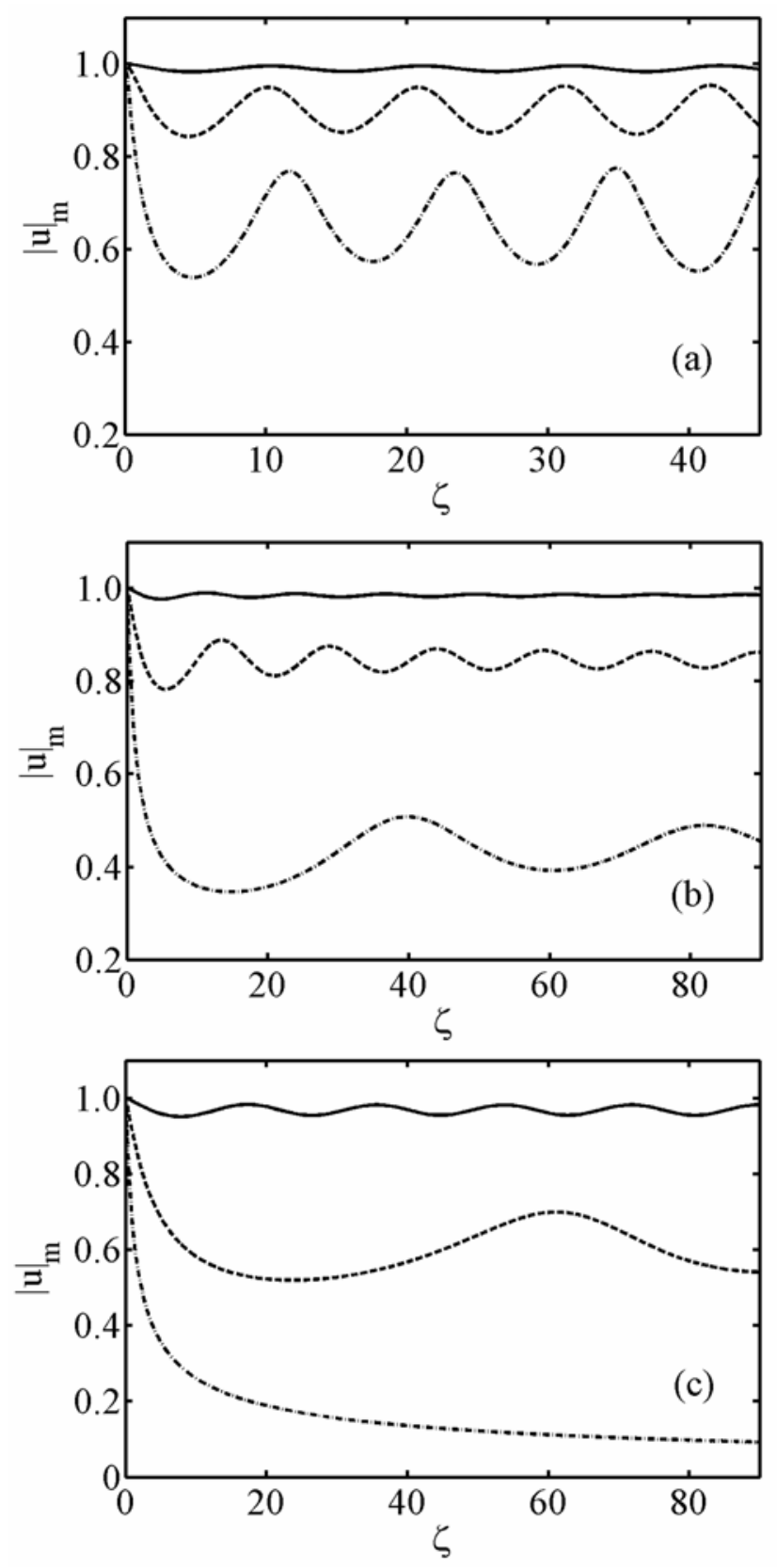
FIGURE 5

J. M. Christian et al.

to appear: Physical Review A
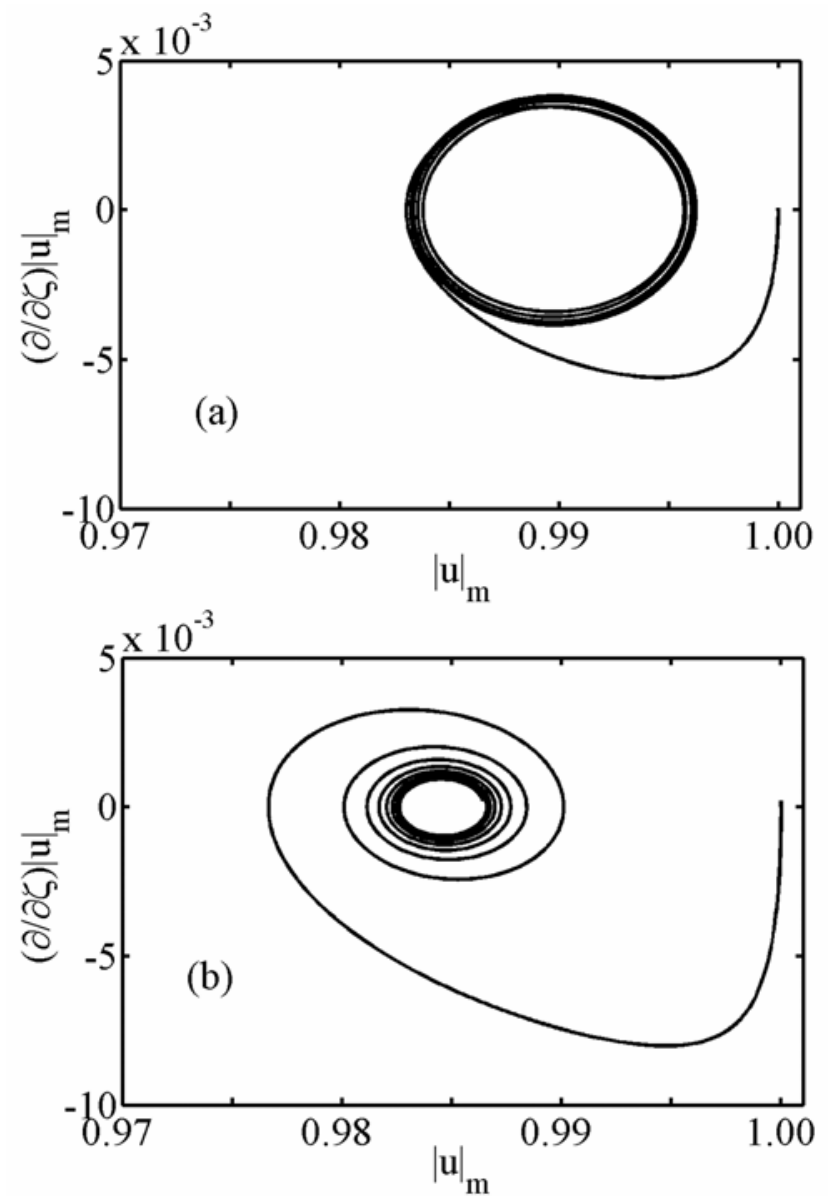
FIGURE 6

J. M. Christian et al.

to appear: Physical Review A

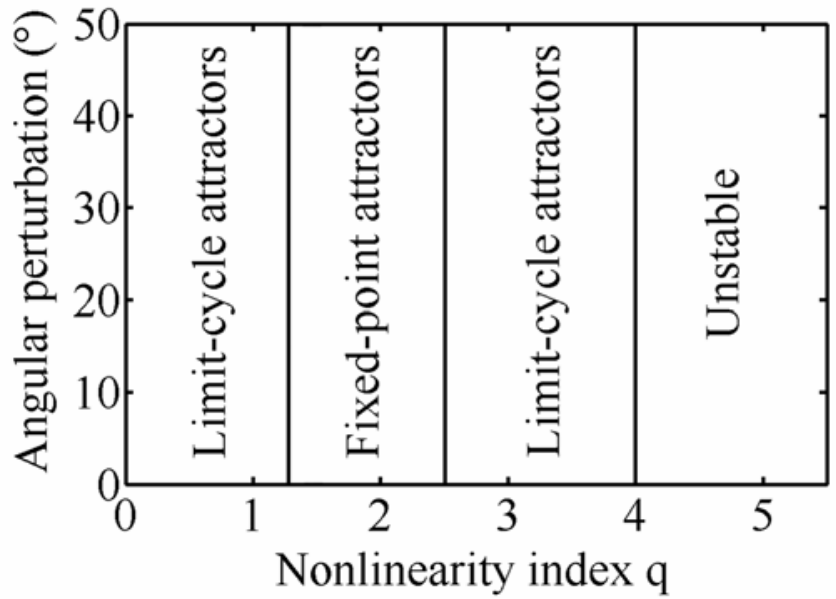


FIGURE 7

J. M. Christian et al.

to appear: Physical Review A

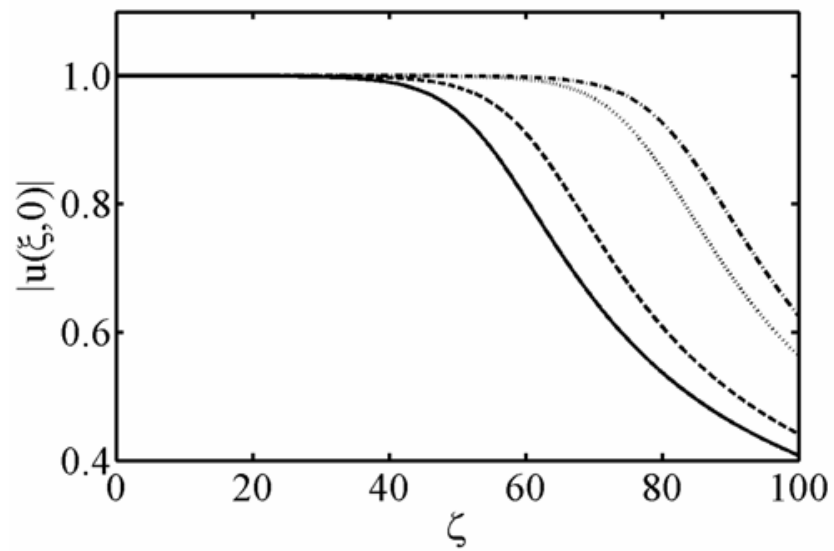

